\title{
LA COMPETENCIA COMUNICATIVA EN PERSONAS QUE PRESENTAN PARÁLISIS CEREBRAL
}

\section{COMMUNICATIVE COMPETENCE IN PEOPLE WITH CEREBRAL PALSY}

ROSADO AYALA, J. I. (jira188@gmail.com). Universidad de Granada

Fecha de recepción: 28 de mayo de 2012

Fecha de aceptación: 11 de junio de 2012

Localizador: http:www.ugr.es/local/miguelgr/ReiDoCrea-Vol.1-Art.21-Rosado.pdf

\section{RESUMEN}

A pesar de los numerosos intentos por definir universalmente la Parálisis Cerebral, todavía no se ha conseguido una definición que una todas las opiniones acerca de ésta, aunque sí existen puntos en común en la mayoría de ellas.

Todas las personas, para poder desenvolverse en su contexto social, deben desarrollar su capacidad de comunicación, especialmente las personas con parálisis cerebral. Esta capacidad se debe potenciar, desde el desarrollo de la lengua por parte de los centros educativos, a través del desarrollo de la competencia comunicativa ya sea a través del lenguaje oral o a través de los sistemas alternativos/aumentativos de comunicación.

Palabras clave: discapacidad, persona con discapacidad, comunicación, comunicación alternativa, competencia comunicativa.

\section{ABSTRACT}

Despite numerous attempts to universally define Cerebral Palsy, there is still no definition that includes all opinions about this, although there are common points in most of them.

All persons in order to function in their social context must develop their communication skills, especially people with cerebral palsy. This capacity must be strengthened, by the development of language in schools, by means of the development of communicative competence either through speech or through alternative systems / augmentative communication.

Keywords: disability, disabled person, communication, alternative communication, communicative competence. 


\section{INTRODUCCIÓN}

Como indican diversos autores, la Parálisis Cerebral es la causa más frecuente de discapacidad motora en la edad pediátrica (Robaina, Riesgo y Robaina, 2007) y (Romeu, Canosa y Gómez Taibo, 2010). La Parálisis Cerebral se ha convertido en ese gran cajón desastre en el que se integran una gran cantidad de afectaciones diferentes, y que a su vez no tiene entidad propia a nivel de reconocimiento como una de las grandes afectaciones, ya que está integrada dentro de la discapacidad motora.

Tras los múltiples intentos por definir la Parálisis Cerebral Infantil, se mantiene todavía abierta las diferencias entre los distintos autores. Aún así, se pueden extraer puntos en común que en cierta manera establece la columna vertebral de cualquier definición que pretenda ser rigurosa.

En relación a la comunicación, Lomas, Tusón y Osoro (1996) consideran que en una situación comunicativa se emplean todas las competencias que conforman la competencia comunicativa.

Estas mismas competencias son las que desarrollan todas las personas que presentan Parálisis Cerebral, aunque tengan que recurrir al empleo de sistemas alternativos/aumentativos de comunicación.

\section{DESARROLLO}

La gran cantidad de afectaciones que conforman la Parálisis Cerebral (PC) es uno de los motivos por los que no se cuenta con una definición clara y universalmente aceptada. Otro de los motivos por lo que existen múltiples definiciones de la Parálisis Cerebral Infantil (PCl) es que ninguna de ellas ha conseguido unificar los criterios de los especialistas, aunque, eso sí, se pueden establecer varias características de esta afectación que podrían conseguir el consenso de todos (Camacho-Salas, PallásAlonso, de la Cruz-Bértolo, Simón-de las Heras y Mateos-Beato, 2007), (Robaina, Riesgo y Robaina, 2007) y (Poó, 2008): Se plantea que la PCl es una lesión permanente y no evolutiva, que se produce cuando el cerebro está en desarrollo, que está localizada en el Sistema Nervioso Central y que provoca una alteración en el tono y la respuesta muscular. También existe consenso en afirmar que la $\mathrm{PCl}$ puede traer consigo diferentes afectaciones a nivel cognitivo y/o social.

Esta situación provoca que existan múltiples intentos por establecer una definición universal como la que nos presenta Lorente (2007): "Trastorno del desarrollo del tono postural y del movimiento de carácter persistente (aunque no invariable), que condiciona una limitación en la actividad, secundario a una agresión no progresiva, a un cerebro inmaduro. En la parálisis cerebral el trastorno motor estará acompañado frecuentemente de otros trastornos (sensitivos, cognitivos, lenguaje, perceptivos, conducta, epilepsia) cuya existencia o no, condicionará de manera importante el pronóstico individual de estos niños." ... o como la realizada por Bax, Goldstein, Rosenbaum, Leviton, Paneth, Dan, et al. (2005), en Camacho-Salas, Pallás-Alonso, de la Cruz-Bértolo, Simón-de las Heras y Mateos-Beato (2007) en la que la consideran como "un grupo de trastornos del desarrollo del movimiento y de la postura, que causan limitación en la actividad y son atribuidos a alteraciones no progresivas que ocurren en el cerebro en desarrollo del feto o del niño pequeño; el trastorno motor se acompaña con frecuencia de alteraciones de la sensibilidad, cognición, comunicación, percepción, comportamiento y/o crisis epilépticas." 
Por otro lado, hay que recordar que en España unos 1.500 niños nacen o desarrollan PC cada año, situándose la prevalencia global de esta afectación en torno al 2- 2,5 por cada 1000 recién nacidos vivos en los países desarrollados, según Lleras, Cordovilla y de la Iglesia (2005).

Según Rye, y Skjorten (1990), la comunicación significa ante todo compartir. Compartimos e intercambiamos sentimientos, pensamientos, opiniones 0 informaciones mediante códigos y símbolos que todos los interlocutores pueden entender y manejar.

Para poder comunicarnos, los seres humanos recurrimos a nuestra competencia comunicativa. Éste término fue acuñado, como nos indica Pilleux (2001), por Hymes (1971, 1972 y 1974), aunque fue Fishman (1970) el que se acerca más al concepto al expresar que todo acto comunicativo entre dos o más personas en cualquier situación de intercambio está regido por reglas de interacción social.

Por su parte, Lomas, Tusón y Osoro (1996) consideran que en un encuentro comunicativo, los participantes deben poner en funcionamiento múltiples competencias que abarcan todos esos factores y que constituyen su competencia comunicativa. Estos mismos autores recogen una cita de Cots y otros (1990) donde entienden la competencia comunicativa como la capacidad de saber qué decir, a quién, cuándo y cómo decirlo y cuándo callar.

También entienden la competencia comunicativa como el conocimiento del hecho lingüístico y de los códigos no verbales y de las condiciones de uso en función de los contextos y situaciones de comunicación y del diverso grado de planificación y formalización de esos usos concretos. En esta línea Canale y Swain (1980) en Roldán 2003-2004) establecen diversas competencias necesarias para el desarrollo de la competencia comunicativa, especificando la competencia lingüística, la competencia sociolingüística, la competencia discursiva y la competencia estratégica. Por su parte, Pilleux (2001) coincide sólo en las dos primeras al considerar que son la competencia lingüística, la competencia sociolingüística, la competencia pragmática y la competencia psicolingüística las que forman la competencia comunicativa.

Según Lomas, Tusón y Osoro (1996) se debe entender como finalidad esencial de la enseñanza de la lengua el desarrollo de la competencia comunicativa del alumnado. También nos recuerdan estos autores que, para mejorar las capacidades de uso comprensivo y expresivo que les permita a los usuarios la adquisición de los mecanismos pragmáticos que consolidan la competencia comunicativa de éstos en situaciones concretas de interacción, debemos centrar el interés en algunos aspectos de los enfoques gramaticales que han ido cayendo en el olvido con el paso del tiempo en los centros educativos.

Además de estas indicaciones, es esencial tener en cuenta el amplio abanico de necesidades que pueden presentar las personas afectadas con PCl. Estas alteraciones van desde la afectación más leve, que no afecta al desarrollo normal del lenguaje, hasta la afectación total de los órganos fonadores impidiendo cualquier expresión oral.

En los casos en los que se ve afectada la comunicación oral se debe buscar el desarrollo de los sistemas alternativos/aumentativos de comunicación (SAAC). Estos sistemas pueden dividirse en sistemas que no requieren ayuda, como pueden ser el lenguaje de signos y la palabra complementada, o sistemas que requieren ayuda, como pueden ser el braille, los sintetizadores de voz, el Bliss, el SPC, sistemas pictográficos, aplicaciones informáticas, etc.

160 ReiDoCrea. Revista electrónica de investigación Docencia Creativa. Volumen 1. Páginas 158-163 
Teniendo en cuenta los componentes de la competencia comunicativa que indica Pilleux (2001) se verían afectadas de la siguiente manera:

- En relación a la competencia lingüística, hay que tener en cuenta que, aunque a nivel oral puedan estar afectadas algunas personas, a nivel escrito pueden presentar un nivel de lenguaje adecuado, siempre que la afectación física se lo permita, puesto que la afectación puede afectar la función sonora pero no tiene porqué afectar también la capacidad perceptiva o de procesamiento del lenguaje.

- En relación a la competencia sociolingüística, siempre que su capacidad perceptiva y/o cognitiva no estén alteradas, estas personas mantendrán un nivel adecuado en esta competencia aunque tengan afectados los órganos fonadores.

- En cuanto a la competencia pragmática, las personas con PC que presentan una afectación en el lenguaje oral, cuando han conseguido utilizar un SAAC, mantienen un nivel de esta competencia bastante adecuado, siendo similar al de personas no afectadas por la PC con la misma competencia comunicativa.

- Por último, la competencia psicolingüística que presentan estas personas, al igual que con la competencia pragmática, cuando han conseguido utilizar un SAAC, mantienen un nivel de competencia bastante adecuado, siendo similar al de personas no afectadas por la PC.

En relación a estas competencias, he de añadir que las personas que presentan Parálisis Cerebral y que tienen alguna afectación del lenguaje oral requieren, por lo general, de mayor tiempo para poder expresarse a través de los SAAC o por vía oral si fuera el caso. En definitiva se puede decir que es importante fomentarle la comunicación de cualquier manera posible (Cabrera, 2000). Además, es fundamental contar con el apoyo de la familia en el uso de los SAAC puesto que con ésta se da gran cantidad de intercambios sociales: es fundamental enseñar a la familia a identificar todas las formas en las cuales el niño se puede comunicar (Cabrera, 2000). El papel de la familia es fundamental puesto que es uno de los contextos más importantes del niño o niña, siendo fuente de seguridad y afecto, y más especialmente en el caso del alumnado con necesidades educativas especiales.

En esta línea, Águila y Águila (2009), por su parte, defienden que es fundamental para las personas con discapacidad física desarrollar sus competencias comunicativas tanto para su rehabilitación como para su inclusión social.

\section{CONCLUSIONES}

El tener que recurrir a los SAAC no implica que los objetivos para desarrollar la competencia comunicativa en estas personas tengan que ser muy diferentes. Estas personas, aunque puedan tener afectada su capacidad de expresar oralmente el lenguaje, un gran número de casos no está afectada la capacidad de percibir ni de procesar el lenguaje, por lo que facilitándoles una alternativa en la comunicación, como son los SAAC, y sometiéndoles a entrenamiento para aprender su uso, pueden llegar a comunicarse con otras personas de manera bastante efectiva. En estos casos, y aunque requieran una mayor dedicación de tiempo, se puede comprobar cómo los distintos componentes de la competencia comunicativa se van adaptando al sistema

161 ReiDoCrea. Revista electrónica de investigación Docencia Creativa. Volumen 1. Páginas 158-163 
de comunicación pero se siguen desarrollando paralelamente al de otras personas con capacidades comunicativas similares.

Por otro lado, y teniendo en cuenta las conclusiones que hacen Penniton, Goldbart, y Marshall (2008) sobre la implicación de su estudio sobre la práctica, se puede afirmar que, puesto que "no se mostró que el tratamiento no sea efectivo", debemos seguir en el empeño de contribuir al desarrollo y la mejora de la competencia lingüística en estas personas.

\section{BIBLIOGRAFÍA}

Águila Rojas, Oscar E. y Águila Coghlan, Juan C. (2009). La Teoría de la Comunicación y el desarrollo de competencias comunicativas en las personas con discapacidades físicas. Diálogos de la Comunicación, 78, 1-14.

Cabrera, Jesús J. (2000). Fomento del Desarrollo del Niño con Parálisis Cerebral. Guía para los que trabajan con niños Paralíticos Cerebrales. Organización Mundial de la Salud.

Camacho-Salas, A.; Pallás-Alonso, C. R.; de la Cruz-Bértolo, J.; Simón-de las Heras, R. y Mateos-Beato F. (2007). Parálisis Cerebral: Concepto y Registros de Base Poblacional. Revista de Neurología, 45 (8), 503-508.

Lomas, Carlos; Tusón, Amparo y Osoro, Andrés (1996). Ciencias del lenguaje, competencia comunicativa y enseñanza de la lengua. Signos. Teoría y Práctica de la Educación, 7, 27-53.

Lorente, Isabel (2007). La parálisis cerebral: Actualización del concepto, diagnóstico y tratamiento. Pediatría Integral, XI (8), 687-698.

Lleras, Siro; Cordovilla, Ana y de la Iglesia, Purificación (coords.). (2005). Guía para el seguimiento de niños con Parálisis Cerebral en Atención Primaria. Gerencia Regional de Salud-Junta de Castilla y León.

Pennington, L; Goldbart, J y Marshall, J. (2008). Tratamiento del habla y el lenguaje para mejorar las habilidades de comunicación de niños con parálisis cerebral. Biblioteca Cochrane Plus, 2.

Pilleux, Mauricio (2001). Competencia comunicativa y análisis del discurso. Estudios Filológicos, 36, 143-152.

Poó, Pilar (2008). Parálisis Cerebral Infantil. Protocolos Diagnósticos Terapéuticos de la Asociación Española de Pediatría. Barcelona.

Robaina, Gerardo R.; Riesgo, Solangel de la C. y Robaina, Martha S. (2007). Evaluación diagnóstica del niño con parálisis cerebral. Revista Cubana de Pediatría, 79 (2).

Roldán, Eduardo (2003-2004). La competencia comunicativa y la expresión oral. Documentos Lingüísticos y Literarios, 26-27, 31-32.

Romeu, Tania; Canosa, Nereida M. y Gómez Taibo, Mà Luisa (2010). Estudio descriptivo del uso de productos de apoyo para la comunicación escrita empleada por alumnos con parálisis cerebral escolarizados en el Área Metropolitana de A Coruña. En Arnaiz, P.; Hurtado, M. D. y Soto, F. J. (Coords.) 25 Años de Integración Escolar en 
España: Tecnología e Inclusión en el ámbito educativo, laboral y comunitario. Consejería de Educación, Formación y Empleo. Murcia.

Rye, Henning y Skjorten, Miriam D. (1990). Guía para la educación de los niños afectados de parálisis cerebral grave. Cuadernos de Educación Especial № 7. UNESCO. 